Int. J. Dev. Biol. 48: 613-622 (2004)

doi: $10.1387 / \mathrm{ijdb} .041829 \mathrm{sg}$

Original Article

\title{
Additional enhancer copies, with intact cdx binding sites, anteriorize Hoxa-7/lacZ expression in mouse embryos: evidence in keeping with an instructional cdx gradient
}

\author{
STEPHEN J. GAUNT* ${ }^{*}$, ADAM COCKLEY and DEBORAH DRAGE \\ Department of Development and Genetics, The Babraham Institute, Babraham, Cambridge, U.K.
}

\begin{abstract}
Expression of a Hoxa-7/lacZ reporter construct in transgenic mouse embryos is shifted anteriorly when the upstream enhancer is multimerized. The shift occurs in spinal ganglia, neurectoderm and in both paraxial and lateral plate mesoderms. Much of the multimer effect is inhibited by destruction of a single caudal (cdx) binding motif in the additional copies of the enhancer. These observations are in agreement with earlier enhancer multimerization analyses made for Hoxb-8 (Charité et al., 1998). Our findings therefore provide further evidence that the anterior limit of a Hox gene's expression domain is normally dependent upon and is determined by, the dosage of transcription factor(s) which bind to its enhancer element(s) and that these factors may be, or must include, the cdx proteins. We consider these findings in terms of both instructional (morphogen-like) gradient and timing models for the establishment of Hox gene expression domains. Enhancer multimerization results in an earlier onset of Hoxa-7/lacZ activity in the embryo. In neurectoderm at 8.7 days and in mesoderm at 10.5 days, the anterior boundaries of expression are located posterior to those seen at some earlier stages of development. We discuss how these findings are in keeping with a model where Hox expression boundaries become set along instructional cdx gradients, formed by cdx decay in cells moving away from the primitive streak region.
\end{abstract}

KEY WORDS: Hoxa-7/lacZ, transgene, enhancer multimer, $c d x$

\section{Introduction}

It remains uncertain as to how Hox expression boundaries become established during vertebrate embryogenesis (e.g. Gaunt, 2000). In 'instructional (morphogen-like) gradient' models, there is a posterior-to-anterior gradient of Hox-inducer along the embryo, with each Hox gene expression boundary becoming set at its own unique concentration threshold. Graded proteins that could potentially function as such Hox gene inducers include caudal (cdx) (Charité et al., 1998; Gaunt et al., 2003), Wnt3A (Kiecker and Niehrs, 2001; Aulehla et al., 2003) and FgF8 (Dubrulle et al., 2001; Liu et al., 2001). In the alternative 'timing model', boundary positions become established according to the time at which a Hox gene is first expressed in the posterior founder tissues of the embryo: the earlier the expression, the more anterior the eventual boundary position (Dollé et al., 1989). It is quite possible that both models have a role to play and that the relative importance of these roles may differ between neurectoderm and mesoderm. We use the term instructional gradient here because of a trend to confine the definition of morphogen to organizer substances that form their gradients by spreading between cells (e.g., Vincent and Briscoe, 2001). Gradients of cdx proteins are not formed in this way (Gaunt et al., 2003; Gaunt, 2004).

Charité et al. $(1995,1998)$ found that multiple copies of a Hoxb8 enhancer cause a forward shift in the expression boundaries of a Hoxb-8/lacZtransgene. The shift was seen in both neurectoderm and mesoderm. This enhancer dosage-effect must be interpreted differently in terms of the two models mentioned above. The timing model predicts that multiple enhancer copies result in earlier expression of the transgene, thereby generating a more anterior boundary. The instructional (morphogen) gradient model predicts that increased enhancer copies make the transgene more sensitive to the Hox inducing morphogen gradient, thereby setting expression at a more anterior position along the axis. Charité et al. (1998) argued in terms of the second of these two mechanisms and

Abbreviations used in this paper: cdx, caudal; RARE, retinoic acid response element.

\footnotetext{
*Address correspondence to: Dr. Stephen J. Gaunt. Department of Development and Genetics, The Babraham Institute, Babraham, Cambridge, CB2 4AT., U.K. Fax: +44-1223-496022. e-mail: stephen.gaunt@bbsrc.ac.uk
} 
suggested that the transducer of positional information to the Hox genes (the morphogen) may be cdx proteins. In support of this, they produced evidence that $c d x$ protein binding activity is graded in concentration along the embryo, that ectopic expression of cdx-4 is able to activate ectopic transcription of Hoxb-8, and that the Hoxb-8 enhancer dosage-effect depends critically upon an intact cdx protein binding motif.

Other studies have also shown that cdx proteins are upstream regulators of Hox genes and that they have a dose-dependent effect upon the position of Hox expression boundaries (Deschamps et al., 1999; Lohnes, 2003, for reviews). Thus, cdx knockouts may give posterior shifts in Hox gene expression boundaries (Subramanian et al., 1995; Chawengsaksophak et al., 1997; van den Akker et al., 2002; Houle et al., 2003), while cdx overexpressions and mis-expressions may cause anterior shifts (Pownall et al., 1996; Epstein et al., 1997; Charité et al., 1998; Ehrman and Yutzey, 2001; Bel-Vialar et al., 2002). Posterior-to-anterior gradients of cdx gene products have been shown in both protein immunohistochemistry (Gamer and Wright, 1993; Meyer and Gruss, 1993) and $c d x /$ lacZ reporter studies (Gaunt et al., 2003). These gradients apparently arise by decay of cdx activity (or by decay of an upstream regulator of cdx activity) as, during gastrulation, cells move anterior to the vicinity of the primitive streak to populate the growing neurectoderm and mesoderm germ layers (Gaunt et al., 2003).
In the present paper, we examine the effect of enhancer dosage on the expression boundaries of a Hoxa $7 / / a c Z$ transgene. The upstream enhancer element of the Hoxa-7gene has been identified in previous studies (Püschel et al., 1991; Knittel et al., 1995; Gaunt et al., 1999; Kim et al., 2002). We now show that adding three extra copies of the enhancer to a Hox/lacZreporter causes a forward shift in its expression within spinal ganglia, neurectoderm and both paraxial and lateral plate mesoderms. Much of this effect depends upon the integrity of a single cdx protein binding motif. Enhancer multimerization also results in an earlier onset of Hox/lacZ activity. However, this change in timing may not be responsible for the forward shift in Hox/lacZ expression.

\section{Results}

\section{Multimerization of the Hoxa-7 upstream enhancer element causes forward shift in Hoxa-7/lacZ expression}

We earlier reported a chick Hoxa-7/lacZ reporter construct (construct 1 in Fig. 1) that is expressed in embryos of a transgenic mouse line with anterior boundaries at the levels of spinal ganglion 5 (sg5) in neural tissue, and prevertebra 13 (pv13) in paraxial mesoderm (Gaunt 2001; Fig. 2 A,H,I). In Fig. 2, boundary positions in paraxial mesoderms are also given (in brackets) as somite (s) addresses. Pv13 corresponds to s17/18 (Burke et al., 1995).

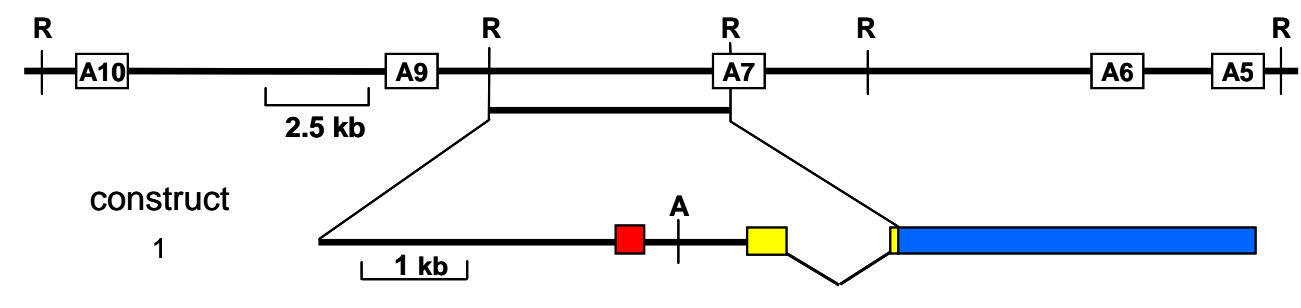

2
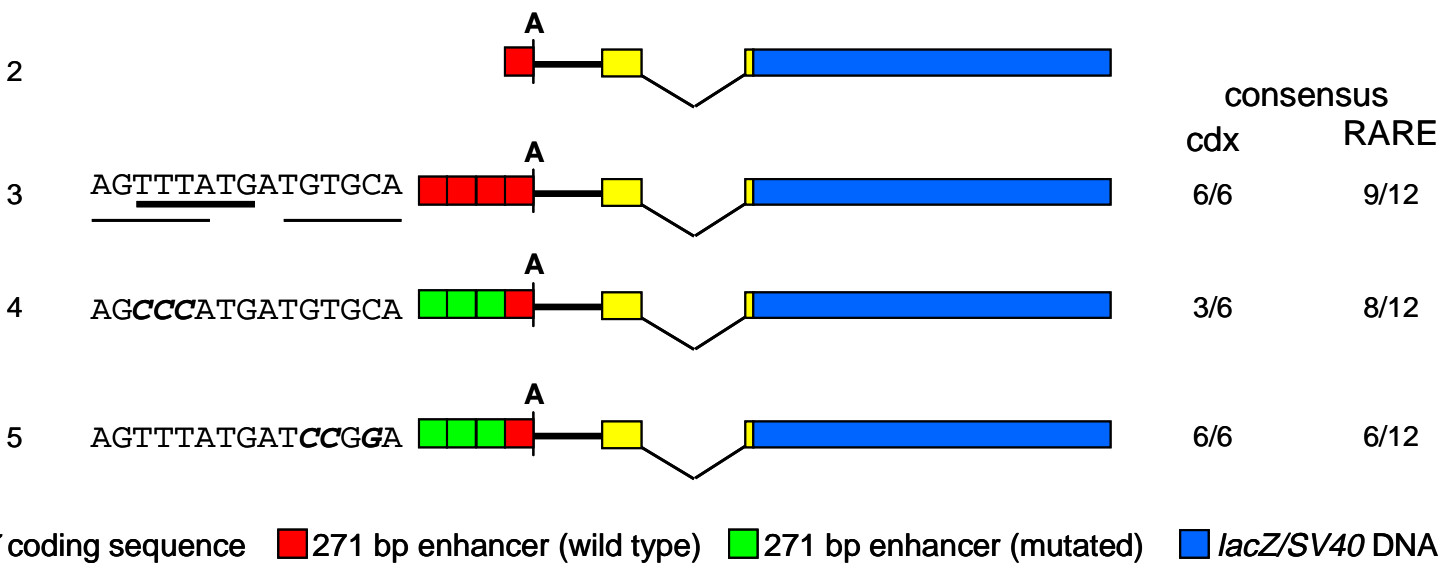

Hoxa-7 coding sequence $\square 271$ bp enhancer (wild type) $\square 271$ bp enhancer (mutated) $\square$ lacZ/SV40 DNA

Fig. 1. Constructs used in the production of Hoxa-7/lacZ transgenic embryos. The chick Hoxa map and construct 1 are as shown by Gaunt et al. (1999). Construct 2 has one normal copy of the 271 bp enhancer region. Construct 3, with four normal copies of the enhancer region, shows the wild-type sequence of the cdx binding motif (thick underline; Margalit et al., 1993) and the repeats of the putative DR3-type RARE (thin underline; Kim et al., 2002). The lower constructs resemble construct 3 except that they are mutated at the bases shown in bold italics, within either the cdx motif (construct 4) or the RARE (construct 5) of the three additional copies of the enhancer fragment. 'Consensus' shows the fraction of the bases within either the cdx binding motif or the RARE repeats that are in accordance with the consensus sequences, as defined in Margalit et al. (1993, for cdx) and Chambon (1994, for RARE). The consensus sequence for a RARE consists of direct repeats of PuGG/TTCA, usually spaced by either two or five nucleotides. R, EcoR1, A, Apa1. 

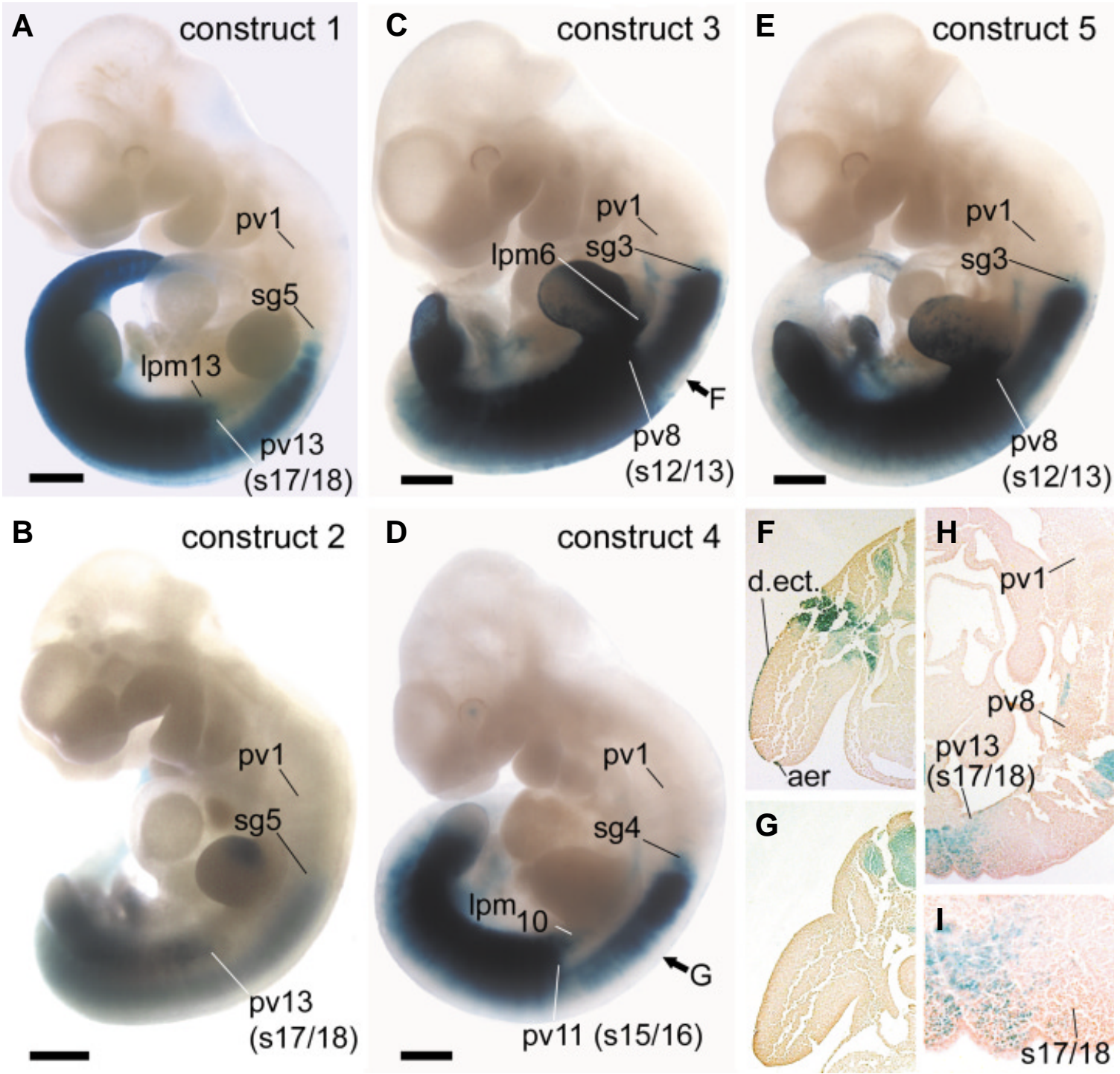

Fig. 2. Constructs $\mathbf{1}$ to $\mathbf{5}$ expressed in the $\mathbf{1 0 . 5}$ day mouse embryo. Four copies of the enhancer unit (construct 3) (C) anteriorizes the expression boundaries in spinal ganglia and mesoderm relative to a single copy (constructs 1 and 2 (A,B) respectively). Much of this anteriorization is lost after mutation of the cdx binding motif within the additional copies of the enhancer unit (construct 4) (D), but not after mutation within a putative RARE (construct 5) (E). Boundaries in paraxial mesoderm are shown both as prevertebral (pv) and somite (s) addresses, as in Burke et al. (1995). Boundaries in lateral plate mesoderm are labelled relative to prevertebral position. Abbreviations: aer, apical ectodermal ridges; d.ect., dorsal ectoderm; sg, spinal ganglion; Ipm, anterior boundaries in lateral plate mesoderm. Arrows in (C,D) mark the positions of the transverse sections shown in (F,G) respectively. A parasagittal section through the embryo shown in (A) is presented in low (H) and high (I) power views. Bars, $0.5 \mathrm{~mm}$.

Three transient transgenic embryos showed similar neural expression boundaries and boundaries in paraxial mesoderm that varied from pv12 to pv13 (Gaunt et al., 1999).

The upstream enhancer of Hoxa-7 has been characterized both structurally and functionally (Knittel et al., 1995). It is seen as a region of sequence homology when chick and mouse (Gaunt, 2001) or chick and human (Fig. 3) DNAs are compared. To make construct 2 (Fig. 1) much of the upstream sequence of construct 1 is replaced with the 271 bp conserved fragment shown in brackets in Fig. 3. In transgenic mouse embryos, construct 2 is expressed with the same anterior boundaries as construct 1 in both spinal ganglia and mesoderm tissues (Fig. 2B). This same observation was made in a second, independently-derived transient transgenic embryo (not shown). The embryo shown also has lacZ activity in the anterior part of the forelimbs, but we attach no significance to this finding since the full-length construct (construct 1) also produces lacZ expression in the anterior forelimb in a proportion of independently produced transgenic embryos (Gaunt et al., 1999).

Construct 3 (Fig. 1) differs from construct 2 in that it contains four identical copies of the $271 \mathrm{bp}$ fragment, arranged in tandem. When analysed in embryos from a transgenic line, this multimerization of the enhancer element results in clear anterior shifts in expression (Fig. 2C). In three independently-derived 
transgenic embryos (two of which are not shown), the anterior boundary in spinal ganglia varied from sg2 to $\mathrm{sg} 3$ and the boundary in paraxial mesoderm was at the level of pv8 (s12/13). The boundary in paraxial mesoderm was therefore located two vertebra positions anterior to the posterior edge of the forelimb. In contrast, the corresponding boundary for constructs 1 and 2 is located three vertebra positions posterior to the forelimb (Fig. 2 $A, B)$. Construct 3 expression, unlike that of constructs 1 and 2 , extends into the flank over the posterior part of the forelimb. This appears as an extension of the staining in lateral plate mesoderm derivatives, up to the level of pv $6(s 10 / 11)$. This is anterior to the corresponding boundary of construct 1 , located at the level of pv13 (s17/18). Within the forelimb, construct 3 is expressed in the posterior AER (Fig. 2C) and the dorsal ectoderm (Fig. 2F).

\section{Much of the multimer effect depends upon an intact cdx protein binding motif}

Construct 4 differs from construct 3 in that the three additional copies of the $271 \mathrm{bp}$ fragment are each mutated in the $\mathrm{cdx}$ binding motif as shown in Fig 1. In a transgenic line (Fig. 2D) this resulted in a posterior shift from the expression boundaries reached by

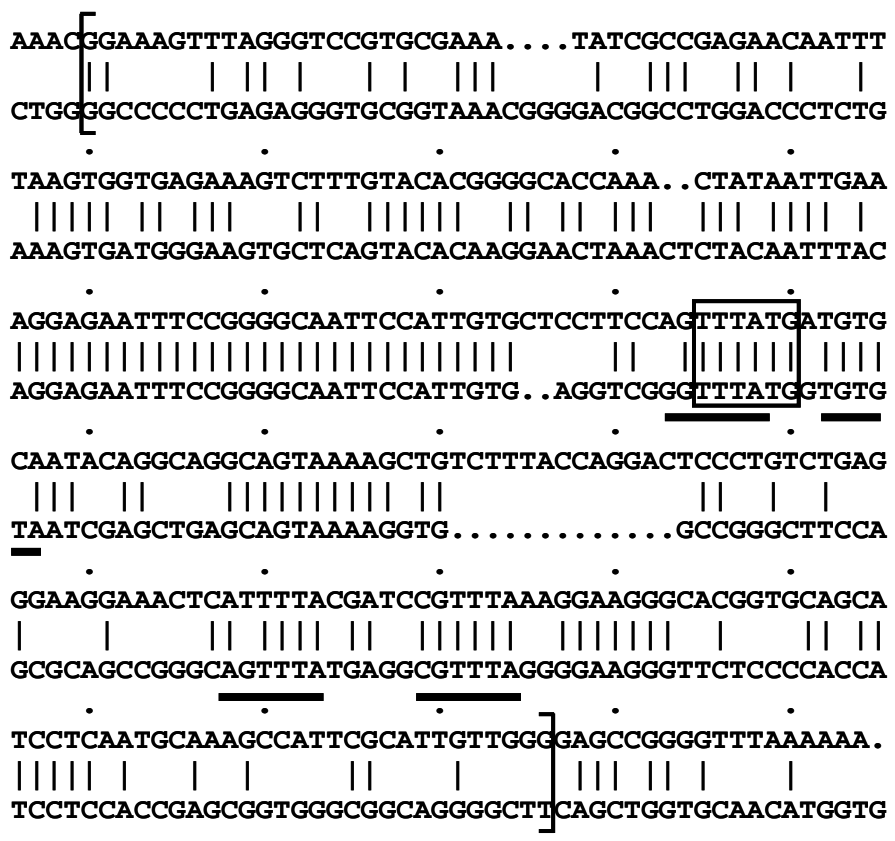

Fig. 3. The upstream enhancer element of Hoxa-7 compared in chick (upper sequence) and human (lower sequence). The region in brackets shows the 271 bp fragment of chick DNA used in the production of constructs 2 to 5 (Fig. 1). The cdx protein binding motif (Margalit et al., 1993), first identified for Hoxa-7 by Subramanian et al. (1993), is shown boxed. Repeats of the putative DR3 and DR5 retinoic acid response elements (Kim et al., 2002) are underlined. Deletion of the DR5 element does not change the position of the neural lacZ expression boundary generated in transgenic mice (Knittel et al., 1995; Kim et al., 2002). The sequences, from EMBL accessions AJ291729 (chick) and AC004080 (human), were compared using Gap from the GCG analysis package (gap weight, 5.0; length weight, 3.0; average match, 1.0; average mismatch, 0.0). The chick sequence shown commences 1227 bp upstream of the coding region.
TABLE 1

\section{SUMMARY OF ANTERIOR BOUNDARY POSITIONS FOR HOXa-7/ LACZ EXPRESSION}

\begin{tabular}{llll} 
& \multicolumn{2}{c}{ Somite address of anterior boundary position } \\
& Construct 1 & Construct 3 & Construct 4 \\
\hline $\begin{array}{l}\text { Neurectoderm } \\
8.25 \text { days }\end{array}$ & $5 / 6$ (Fig. 4B) & 5 (Fig. 4E) & 5 (Fig. 4H) \\
$\begin{array}{l}\text { 8.7 days } \\
\text { Paraxial mesoderm }\end{array}$ & 7 (Fig. 5 A,A') & 5 (Fig. 5B) & 6 (Fig. 5C) \\
$\begin{array}{l}\text { 8.7 days } \\
10.5 \text { days }\end{array}$ & 14 (Fig. 5 A',D) & 11 (Fig. 5B') & 12 (Fig. 5C') \\
$\begin{array}{l}\text { Lateral plate mesoderm } \\
8.7 \text { days }\end{array}$ & 13 (Fig. 5A') & 10 (Fig. 5B') & 10 (Fig. 5C') \\
10.5 days & $17 / 18$ (Fig. 2A) & $10 / 11$ (Fig. 2C) & $14 / 15$ (Fig. 2D) \\
\hline
\end{tabular}

construct 3 (Fig. 2C). This posterior shift is greater within paraxial mesoderm (where it is three segments) and lateral plate mesoderm (four segments) than in spinal ganglia (one segment). Six independent transient transgenic embryos (not shown) had similar boundaries in spinal ganglia and had mesodermal boundaries that varied from pv10 to pv11 (paraxial mesoderm) and pv9 to pv10 (lateral plate mesoderm). Construct 4, unlike construct 3 , is not expressed in any part of the forelimb (Fig. 2G). The expression boundaries for construct 4 remain, however, slightly anterior to those of constructs 1 and 2 .

These results indicate that much, although not all, of the forward shift due to enhancer multimerization may be nullified by destruction of a single $\mathrm{cdx}$ binding motif within each of the three extra copies of the enhancer. A potential problem with this conclusion, however, is that this cdx binding motif forms part of a putative retinoic acid response element (RARE) (Fig. 3). This is the first and least RA-responsive, of the two RAREs described by Kim et al. (2002). The posterior shift of construct 4 expression relative to construct 3 might therefore be due to destruction of a RARE rather than a cdx binding motif. To distinguish between these possibilities, a new construct was made in which a mutation was introduced to disrupt the RARE but not the cdx motif. Construct 5, containing this mutation in the three additional copies of the $271 \mathrm{bp}$ fragment (Fig. 1), shows identical anterior boundaries of lacZ activity in spinal ganglia and mesoderm (Fig. 2E) as those generated by construct 3 (Fig. 2C). This same result was obtained in three additional transient transgenic embryos (not shown). Overall, therefore, these results indicate no evidence for a functional RARE at this position and show instead that the forward shift in lacZ expression caused by multimerization of the $271 \mathrm{bp}$ element requires the intact $\mathrm{cdx}$ protein binding motif.

\section{Effect of multiple cdx binding sites on the timing of initial expression}

Transgenic lines expressing either constructs 1, 3 or 4 were compared in their times of initial expression. Construct 3 (Fig. 4D) commenced expression at the early headfold stage, while constructs 1 (Fig. 4A) and 4 (Fig. 4G) commenced expression later, at the late headfold stage. In all cases, this initial expression was mainly confined centrally to the primitive streak. It therefore appears that multiple copies of the enhancer increase the sensi- 

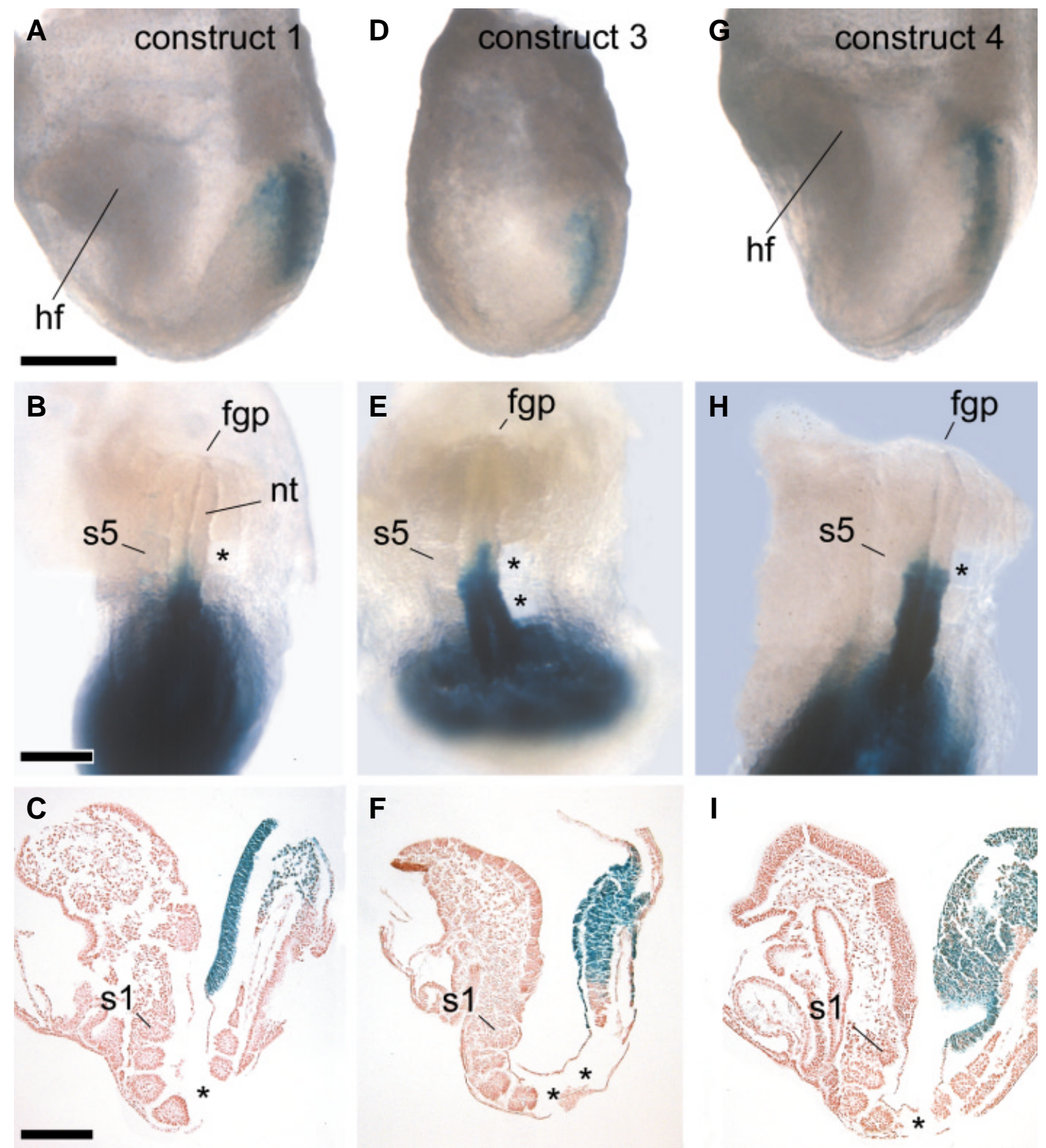

Fig. 4. Early expression of constructs $\mathbf{1}, \mathbf{3}$ and 4. For each construct, expression is shown at the stage of its earliest detection (A,D,G) and also at 8.25 days $(\mathbf{B}, \mathbf{C}, \mathbf{E}, \mathbf{F}, \mathbf{H}, \mathbf{I})$. The lines of mice examined are the same as those shown in Fig. 2 A, C,D. The positions of the expression boundaries in neurectoderm at 8.25 days $(B, E, H)$ were assessed by removal of adjacent somites (asterisks), followed by location of the gaps in parasagittal sections (C, $F, I$, respectively). Abbreviations: fgp, foregut pocket; $h f$, headfold; $n t$, neural tube; s, somite. Bars, $0.25 \mathrm{~mm}$.

tivity of the transgene to transcription factors, causing earlier expression, but that this effect is not seen if the extra copies are debilitated by destruction of the cdx binding site. We considered the possibility that constructs 1 and 4 commence expression at the early headfold stage, but at much lower levels than construct 3. While this possibility is difficult to rule out, we were unable to detect even low levels of lacZ staining before late headfold stage (not shown). Constructs 1 and 4 did not appear to be expressed any less intensely than construct 3 at later stages.

\section{Early expression of Hoxa-7/lacZ extends anterior to its defini- tive boundaries}

At the 9 somite stage (8.25 days), construct 1 expression has an anterior boundary in neurectoderm at the level of the junction between somites 5 and 6 (Fig. 4B). This somite address was identified by removing an adjacent somite (Fig. 4B) and then locating the initial position of this extracted somite in parasagittal sections (Fig. 4C; see Materials and Methods). At this same stage, constructs 3 (Fig. 4 E,F) and 4 (Fig. 4 H,I) showed 

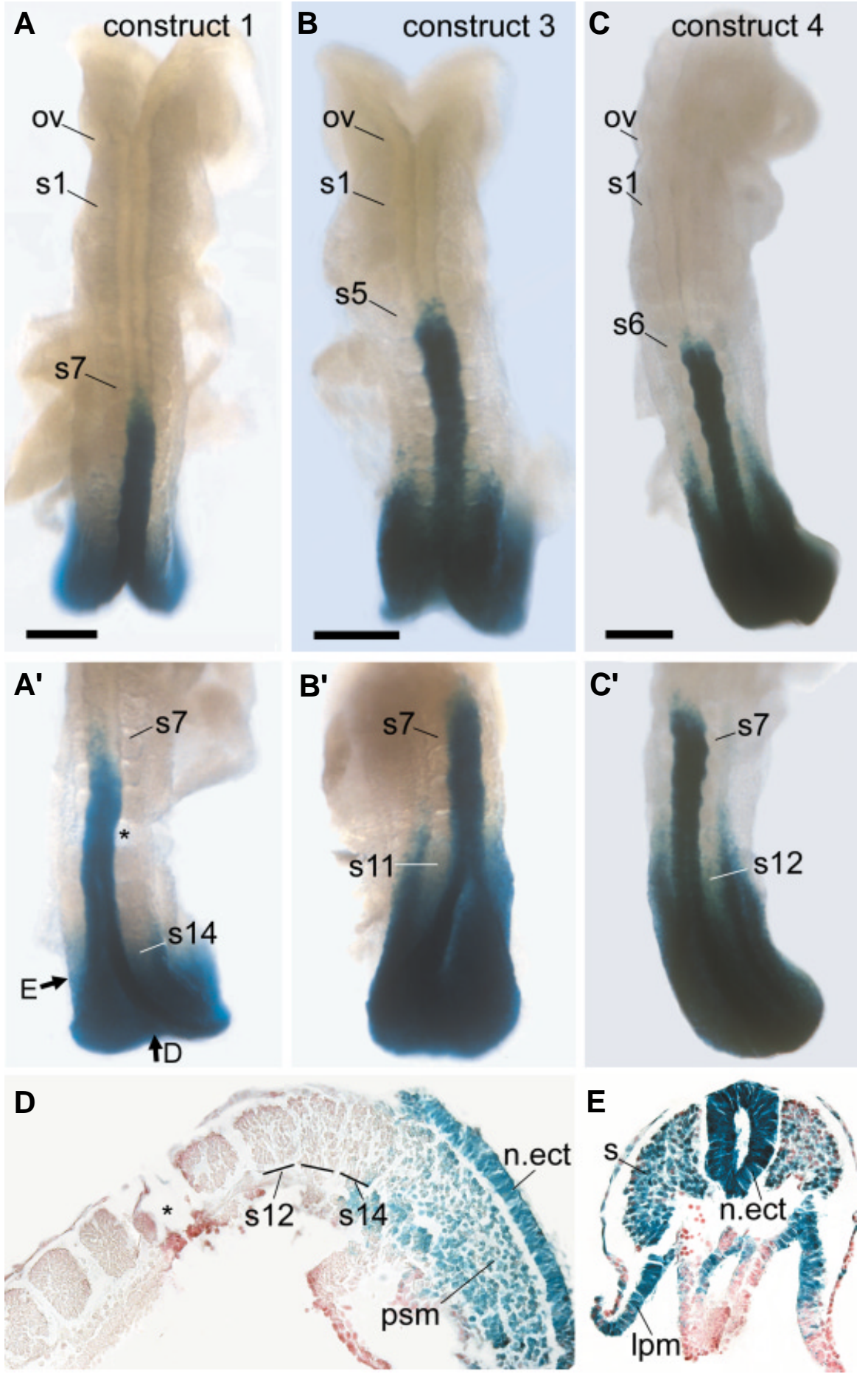

Fig. 5. Constructs $\mathbf{1}, \mathbf{3}$ and $\mathbf{4}$ expressed in $\mathbf{8 . 7}$ day mouse embryos. The lines of mice examined are the same as those shown in Figs. 2 A, C, D and 4. The embryos shown in (A, B, C) are positioned below ('A', B', C'), respectively, to better illustrate boundaries in paraxial and lateral plate mesoderms. In $A^{\prime}$ and its accompanying section (D), somite 10 has been removed (asterisk) and arrows show the planes of section illustrated in (D), which is a parasagittal section of embryo $A^{\prime}$, and (E) which is an obliquely transverse section of a similar embryo, also expressing construct 1 . The more weakly staining (right) side of (E) indicates a more anterior position, since lacZ is expressed in a posterior to anterior gradient along the developing paraxial mesoderm (Fig. 5D; Gaunt, 2001). Abbreviations: Ipm, lateral plate mesoderm; n.ect, neurectoderm; ov, otic vesicle; psm, presomitic mesoderm; s, somite. Bars, $0.5 \mathrm{~mm}$.

apparently similar anterior boundaries of neural expression, at levels corresponding to about the middle of somite 5 . These boundaries are located about half a somite level anterior to the neural boundary of construct 1.

In Fig. 5, expression boundaries in neurectoderm and mesoderm are located at 8.7 days (ca. 15 somite stage). The appearance of the otic vesicle at this stage facilitates localization of somite 1 (Theiler, 1989). At 8.7 days, the anterior boundary of construct 1 expression in neurectoderm has clearly shifted posteriorly, to the level of somite 7 (Fig. 5A, A'; c.f. Fig. 4B at 8.25 days). This anterior-to-posterior regression in neural tissue was less evident for constructs 3 and 4 . Hence, the neural boundary for construct 3 remains at about the level of mid somite 5 (Fig. 5 B,B'), while the boundary for construct 4 appears to move posteriorly only slightly, to the level of mid somite 6 (Fig. 5 C, C').

For constructs 1, 3 and 4, anterior boundaries in paraxial mesoderm at 8.7 days are detected in somites 14 (Fig. 5 A',D), 11 (Fig. $\left.5 B^{\prime}\right)$ and 12 (Fig. 5C'). The corresponding boundaries in lateral plate mesoderm are at the levels of somites 13 (Fig. 5A'), 10 (Fig. 5B') and 10 (Fig. 5C'). We conclude that these boundary positions must later regress posteriorly in order to reach the levels seen at 10.5 days (Fig. 2 A,C,D).

Table 1 summarizes the positions of these anterior expression boundaries for constructs 1,3 and 4 in neurectoderm, paraxial and lateral plate mesoderms. The data indicate that there is a time in the early activity of the transgenes when expression over-reaches its eventual boundary position. Subsequent A-P regression of the expression boundary is greatest, in both neural and mesoderm tissues, for the most posteriorly expressed construct (construct 1) and is least for the most anteriorly expressed construct (construct 3).

\section{Discussion}

Extra copies of an upstream enhancer anteriorize Hoxa7/lacZ expression

We show that expression of a Hoxa-7/ lacZ reporter construct is shifted anteriorly when an upstream enhancer element is multimerized and that this occurs in spinal ganglia, neurectoderm, paraxial mesoderm and lateral plate mesoderm. Similar results were earlier obtained for Hoxb-8by Charité etal.(1995, 1998). These findings are readily 
explained if the anterior limits of a Hox gene's expression domain are normally dependent upon and determined by, the dosage of transcription factor(s) that binds to its enhancer element(s). Thus, the binding may be increased (and hence the boundary shifted anteriorly) either by an increased concentration of transcription factor, such as cdx protein (e.g. Epstein et al., 1997), or else by an increase in the number of copies of the enhancer monomer unit.

\section{The role of caudal (cdx) proteins in the regulation of Hox expression boundaries}

Much of the multimer effect is inhibited by destruction of the cdx binding site within the additional copies of the Hoxa-7enhancer element. Charité et al. (1998) obtained a similar result for Hoxb8. One possibility is that cdx proteins themselves are the dosedependent regulatory factors which determine Hox expression boundaries. The results do not rule out an alternative possibility that it is some other enhancer-binding factor that is limiting on the setting of Hox boundaries, with cdx being an essential co-factor. However, since increased cdx protein alone is able to anteriorize Hox gene expression (Pownall et al., 1996; Epstein et al., 1997; Charité et al., 1998; Ehrman and Yutzey, 2001; Bel-Vialar et al., 2002) it does seem that cdx proteins themselves are likely to be important regulators of Hox gene expression boundary positions.

\section{Anterior-to-posterior regression of Hox/lacZ activity in neurectoderm and mesoderm}

Table 1 summarizes the positions of anterior expression boundaries observed within both neurectoderm and mesoderm at various times of development. For each tissue, it is seen that the anterior boundary tends to be more anterior at the earlier stages. The subsequent anterior-to-posterior $(A-P)$ regression is more evident for constructs that eventually adopt a more posterior expression boundary (constructs 1 and 4). The regressions that we describe may have a similar mechanism to the regressions noted earlier, by in situ hybridization, for the anterior boundaries of Hoxa-4 in the chick paraxial mesoderm (Gaunt, 2000) and Hoxb-9 in the chick neurectoderm (Bel-Vialar et al., 2002). A related mechanism might account for regression in the expression of a Hoxb-4/lacZtransgene (Brend et al., 2003). A prediction of these observations is that a cell located at the anterior boundary of a Hox gene's expression at an early developmental stage will, at least in the case of some Hox genes, be situated some distance anterior to the boundary as it will exist at a later stage. This prediction is consistent with the mesodermal fate mapping studies of Forlani et al. (2003).

A-P regression of Hox expression boundaries is apparently opposite to the forward spreading that has been described in several earlier papers (Deschamps and Widgerde, 1993; Gaunt and Strachan, 1994, 1996). Forward spreading occurs in certain situations. For example: 1) expression of anterior Hox genes in the neural tube, where forward extension of expression is induced as a secondary event by induction from underlying somites (Itasaki et al., 1996; Grapin-Botton et al., 1997) in a retinoiddependent mechanism (Liu et al., 2001; Oosterveen et al., 2003). 2) During the early phase of a Hox gene's expression along the primitive streak (Deschamps and Wijgerde, 1993; Gaunt and Strachan, 1994, 1996; Forlani et al., 2003). 3) As a forward spread in the abundance of transcripts within an initially graded Hox expression domain (Gaunt, 2001). A possible explanation for A$P$ regression of Hox gene expression boundaries is suggested by the expression pattern of their upstream activators, the cdx proteins. Thus, cdx gene expressions also show A-P regression (Pillemer et al., 1998). We have suggested that this is a feature of the mechanism whereby cdx gene expression gradients are set up along the axis (Gaunt et al., 2003). Our proposal was that cdx proteins are synthesised mainly within the vicinity of the primitive streak and that gradients form by decay of cdx proteins (or their upstream activators, such as Wnt in the case of cdx1; Lickert et al., 2000) within cells once they move anteriorward of the primitive streak region. In support of this, we showed that the $\mathrm{cdx}-1 / \mathrm{lacZ}$ activity gradient in transgenic mice forms in this way, by decay of activity as cells move forward of the primitive streak region in order to contribute to the growing body axis (Gaunt et al., 2003). The time over which we now observe anterior-to-posterior regression of Hox/lacZ activity (8.25 to 8.7 days) shows good correspondence with that over which there is regression and gradient formation in the lacZ activity of $c d x-1 /$ lac $Z$ transgenic embryos (Gaunt et al., 2003).

The forward shift of construct 3 expression into the posterior AER and dorsal ectoderm of the forelimb (Fig. 2F) provides additional evidence for the role of $\mathrm{cdx}$ as activator of the Hoxa-7 enhancer. Thus, these parts of the forelimb are both sites of cdx1 activity, as indicated in $c d x-1 / / a c Z$ reporter embryos (Gaunt et al., 2003).

\section{A timing or an instructional gradient mechanism to set Hox expression boundaries?}

A novel observation in this paper is that manipulations made upon the regulatory elements of a transgene can change not only its anterior limits of expression, but also the time of its initial expression in the embryo. Thus, expression of a transgene with four copies of the enhancer is first detected earlier in time (early headfold stage) than is either a transgene with only one copy, or else is a transgene with one normal copy plus three mutated within a cdx binding motif (late headfold stage). This particular observation means that it is not possible to say with certainty whether the findings reported are best in-keeping with the 'instructional gradient' or 'timing' models (Introduction; see also Gaunt, 2000). Thus, the forward shift in Hox gene expression caused by four enhancer units might be due either to activation earlier in time (timing model) or else to activation at a lower concentration along a posterior-toanterior instructional gradient along the embryo (instructional gradient model).

Certain observations do, however, seem to be more in keeping with the instructional gradient model. For example: 1) Construct 3 shows earlier expression than construct 4, yet both apparently display rather similar anterior boundaries of expression in neurectoderm at 8.25 days (Fig. $4 \mathrm{E}, \mathrm{H}$ ) and in paraxial mesoderm at 8.7 days (Fig. $\left.5 \mathrm{~B}^{\prime}, \mathrm{C}^{\prime}\right)$. 2) Constructs 1 and 3 show neural expression boundaries differing by a distance of only half to one somite at 8.25 days (Fig. 4 B,E), but of two to three somites at 8.7 days (Fig. 5 A,B). 3) Constructs 3 and 4 show paraxial mesoderm expression boundaries differing by only about one somite at 8.7 days (Fig. 5 B',C'), but by the equivalent of three somites at 10.5 days (Fig. 2 C,D).

The findings are consistent with a model (e.g., Fig. 6) in which anterior boundaries of Hox gene expression become positioned 
along a developing $c d x$ protein instructional gradient. A cdx gradient forming by decay (Gaunt et al., 2003) could account for the observed A-P regression of both $c d x$ and Hox expression. As shown in Fig. 6, more anteriorly expressed Hox genes activated at lower concentrations of $\mathrm{cdx}$ (such as construct 3 , with its multiple cdx binding sites) might be expected to show less A-P regression than more posterior Hox genes activated at higher cdx concentrations (e.g., constructs 1 and 4). Although we discuss a cdx gradient here, it is also possible that other Hox-activating transcription factors (e.g., FGF, Pownall et al., 1996; Liu et al., 2001) form gradients as a result of a decay mechanism. A Wnt3A gradient may form by decay within the presomitic mesoderm and it is apparently a fall to a critical threshold level of Wnt3A that provides the signal for somite separation (Aulehla et al., 2003). The formation of gradients by decay may therefore provide a mechanistic link between the parallel processes of segmentation and Hox boundary formation. However, while decay models predict that segmentation occurs at a fixed distance from the primitive streak, they would also predict that the boundaries of

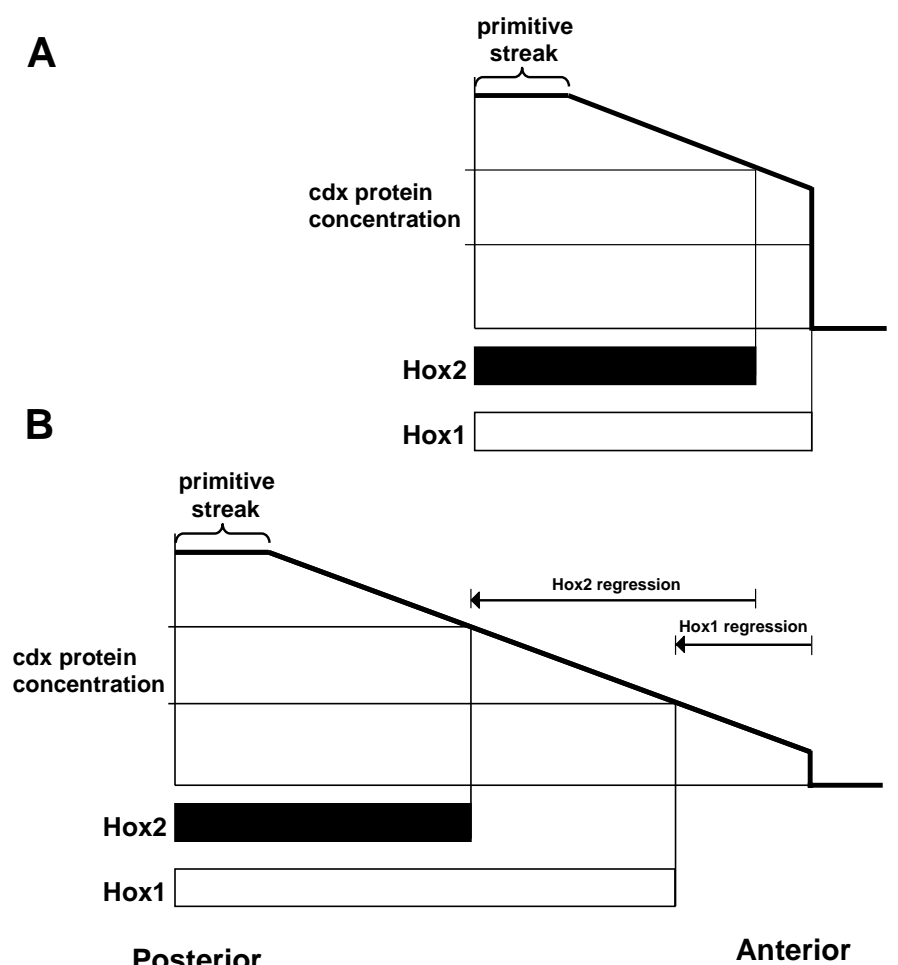

Fig. 6. A scheme for the establishment of Hox expression boundaries along an instructional cdx gradient, formed by decay. With regression of the primitive streak (A to $\mathbf{B}$ ), the $c d x$ protein gradient forms by a process of decay (Gaunt et al., 2003). Expression of the Hox2 gene (Hoxa-7/lacZ reporter with single cdx binding site, or a normal $5^{\prime}$-located Hox gene) is activated at a high threshold concentration of cdx protein. Expression of the Hox1 gene ( Hoxa-7/lacZ reporter with multiple cdx binding sites, or a normal $3^{\prime}$-located Hox gene) is activated at a lower threshold concentration of cdx protein. After time (A to B), the anterior boundaries of Hox expression show anterior-to-posterior regression and this may be greater for more posteriorly expressed genes. The exact kinetics of cdx protein decay is unknown. different Hox genes may form at dissimilar distances from the primitive streak. It is uncertain in this scenario how Hox expression boundaries would eventually become stabilized.

In summary, at least three observations, discussed above, are in keeping with an instructional cdx gradient model for the setting of Hox gene expression boundaries. 1) Hox/lacZ expression boundary positions are apparently determined by the dosage of cdx protein that binds to the Hox enhancer(s) (this paper and Charité et al., 1998). 2) Concentrations of cdx gene products form posterior-to-anterior gradients along the embryo (Gamer and Wright, 1993; Meyer and Gruss, 1993; Charité et al., 1998; Gaunt et al., 2003). 3) There is a good temporal correlation between the time period over which Hoxa-7/lacZ expression boundaries regress along the neural tube ( 8.25 to 8.7 days - this paper) and that over which the $c d x-1 /$ lacZ activity gradient becomes established (Gaunt et al., 2003).

An instructional gradient model can, therefore, accommodate changes in the initial positions of Hox expression boundaries as these evolve during embryonic development. The timing model, on the other hand, predicts that once Hox expression patterns are established in the posterior embryo they become fixed in a lineage-dependent way and it seems less able to accommodate the findings, now described, of changes in the anterior limits of developing Hox gene expression boundaries. Our observations suggest a need for similar studies on other Hox genes, comparing the position of their expression boundaries at early and later stages in gastrulation. The findings can provide support for either the timing or gradient model.

\section{Materials and Methods}

\section{Preparation of chick Hoxa-7/lacZ reporter constructs}

Construct 1 (Fig. 1) has been described earlier (Gaunt etal., 1999). The upstream enhancer element of chick Hoxa-7(Fig. 1,3) was cloned by PCR as a $271 \mathrm{bp}$ fragment using the primers GTCACGAGATCTGGAAAGTTTAGGGTCCGT (5') and TATACAGGGCCCAGTATCATCGGATCCCCAACAATGCGAATGG (3'). This incorporated a Bglll site at the $5^{\prime}$ end and both Apal and BamHI sites at the 3 ' end. After cutting with Bglll and Apal, the fragment was subcloned into the BamHI/Apal sites in the polylinker of pCDNA3 plasmid (Invitrogen). To make construct 2 (Fig. 1), the insert was cut from pCDNA3 using Kpnl and Apal, then used to replace the EcoRI/Apal fragment of construct 1.

Multimers of the Hoxa-7 enhancer were prepared as described by Charite et al. (1998), making use of the fact that Bglll/BamHI ligation sites are not re-cut by BamHI. Thus, the monomer in pCDNA3 was cut with BamHI plus Apal and a second copy of Bglll/Apal cut enhancer fragment was inserted to make a dimer. This process was repeated, producing trimer, then tetramer. The monomer units used at each step were, as required, either normal or contained a defined mutation generated by sitedirected mutagenesis (QuikChange, Stratagene). To make construct 3 (Fig. 1), the tetramer consisted of four normal enhancer monomers. It was cut from pCDNA3 using Kpnl plus Apal, then used to replace the Kpnl/Apal fragment of construct 1. To make construct 4 (Fig. 1), the 3 ' -most monomer was normal, but other units in the tetramer contained a mutation in the cdx binding motif that is known to abolish binding (Charité et al., 1998). To make construct 5 (Fig. 1), the 3 ' -most monomer was normal, but others contained a mutation in the second repeat of the putative RARE. This mutation incorporated a diagnostic Acclll site.

\section{Transgenic embryo production and staining}

This was carried out as described earlier (Gaunt etal., 2003), except that all staining reactions were allowed to proceed to apparent completion (18 
to 30 hours). The embryos shown in Figs. 2 A,C,D 4 and 5 of this paper were from three lines of transgenic mice that express either constructs 1,3 or 4 . Embryos were taken to be at 0.5 days of development at midday on the day of the copulation plug. Transient transgenics, each expressing one of the constructs 2 to 5 , were examined at 10.5 days only.

At stages up to 8.7 days, neural boundaries of Hox/lacZ expression were located relative to somite address. Due to flexure of early embryos ( 8.25 days in this study), somite 1 could not be positively identified in wholemount alone. A special procedure was adopted in order to positively identify somite 1 . We removed one or two somites adjacent to the lacZ expression boundary and then subsequently identified the address of the extracted somites as seen in parasagittal sections (Fig. 4). At 8.7 days, localization of the first somite was facilitated by reference to the position of the newly formed otic vesicle (Theiler, 1989).

\section{References}

AULEHLA, A., WEHRLE, C., BRAND-SABERI, B., KEMLER, R. and HERRMANN, B. G. (2003). Wnt3A plays a major role in the segmentation clock controlling somitogenesis. Developmental Cel/4: 395-406.

BEL-VIALAR, S., ITASAKI, N. and KRUMLAUF, R. (2002). Initiating Hox gene expression in the early chick neural tube: sensitivity to FGF and RA signalling subdivides the HoxB genes in two distinct groups. Development 129: 51035115.

BREND, T., GILTHORPE, J., SUMMERBELL, D. and RIGBY, P. W. J. (2002). Multiple levels of transcriptional and post-transcriptional regulation are required to define the domain of Hoxb4 expression. Development 130: 2717-2728.

BURKE, A. C., NELSON, C. E., MORGAN, B. A. and TABIN, C. (1995). Hox genes and the evolution of vertebrate axial morphology. Development 121: 333-346.

CHAMBON, P. (1994). The retinoid signalling pathway: molecular and genetic analyses. Semin. Cell. Biol. 5: 115-125.

CHARITÉ, J., DE GRAAFF, W., VOGELS, R., MEIJLINK, F. and DESCHAMPS, J. (1995). Regulation of the Hoxb-8 gene: synergism between multimerized cisacting elements increases responsiveness to positional information. Dev. Biol. 171: 294-305.

CHARITÉ, J., DE GRAAFF, W., CONSTEN, D., REIJNEN, M. J., KORVING, J. and DESCHAMPS, J. (1998). Transducing positional information to the Hox genes: critical interaction of $\mathrm{cdx}$ products with position-sensitive regulatory elements. Development 125: 4349-4358.

CHAWENGSAKSOPHAK, K., JAMES, R., HAMMOND, V. E., KÖNTGEN, F. and BECK, F. (1997). Homeosis and intestinal tumours in Cdx2 mutant mice. Nature 386: 84-87.

DESCHAMPS, J., VAN DEN AKKER, E., FORLANI, S., DE GRAAFF, W., OOSTERVEEN, T., ROELEN, B. and ROELFSEMA, J. (1999). Initiation, establishment and maintenance of Hox gene expression patterns in the mouse. Int J. Dev. Biol. 43: 635-650.

DESCHAMPS, J. and WIJGERDE, M. (1993). Two phases in the establishment of Hox expression domains. Dev. Biol. 156: 473-480.

DOLLÉ, P., IZPISUA-BELMONTE, J. C., FALKENSTEIN, H., RENUCCI, A. and DUBOULE, D. (1989). Coordinate expression of the murine Hox-5 complex homeobox-containing genes during limb pattern formation. Nature 342: 767772.

DUBRULLE, J., McGREW, M. J. and POURQUIE, O. (2001). Fgf signalling controls somite boundary position and regulates segmentation clock control of spatiotemporal Hox gene activation. Cel/ 106: 219-232.

EHRMAN, L. A. and YUTZEY, K. E. (2001). Anterior expression of the caudal homologue $\mathrm{cCdx}-\mathrm{B}$ activates a posterior genetic program in avian embryos. Dev. Dyn. 221: 412-421.

EPSTEIN, M., PILLEMER, G., YELIN, R., YISRAELI, J. K. and FAINSOD, A. (1997). Patterning of the embryo along the anterior-posterior axis: the role of the caudal genes. Development 124: 3805-3814.

FORLANI, S., LAWSON, K. A. and DESCHAMPS, J. (2003). Acquisition of Hox codes during gastrulation and axial elongation in the mouse embryo. Development 130: 3807-3819.
GAMER, L. W. and WRIGHT, C. V. E. (1993). Murine Cdx-4 bears striking similarities to the Drosophila caudal gene in its homeodomain sequence and early expression pattern. Mech. Dev. 43: 71-81.

GAUNT, S. J. (2000). Evolutionary shifts of vertebrate structures and Hox expression up and down the axial series of segments: a consideration of possible mechanisms. Int. J. Dev. Biol. 44: 109-117.

GAUNT, S. J. (2001). Gradients and forward spreading of vertebrate Hox gene expression detected by using a Hox/lacZ transgene. Dev. Dyn. 221: 26-36.

GAUNT, S. J. (2004). Morphogen gradients formed by decay. BioEssays 26: 11431144.

GAUNT, S. J. and STRACHAN, L. (1994). Forward spreading in the establishment of a Hox expression boundary: the expression domain separates into anterior and posterior domains and the spread occurs across implanted glass barriers. Dev. Dyn. 99: 229-240.

GAUNT, S. J. and STRACHAN, L. (1996). Temporal colinearity in expression of anterior Hox genes in developing chick embryos. Dev. Dyn. 207: 270-280.

GAUNT, S. J., DEAN, W., SANG, H. and BURTON, R. D. (1999). Evidence that Hoxa expression domains are evolutionarily transposed in spinal ganglia and are established by forward spreading in paraxial mesoderm. Mech. Dev. 82: 109-118.

GAUNT, S. J., DRAGE, D. and COCKLEY, A. (2003). Vertebrate caudal gene expression gradients investigated by use of chick cdx-A/lacZ and mouse $c d x-$ $1 /$ lacZ reporters in transgenic mouse embryos: evidence for an intron enhancer. Mech. Dev. 120: 573-586.

GRAPIN-BOTTON, A., BONNIN, M-A. and LE DOUARIN, N. M. (1997). Hox gene induction in the neural tube depends on three parameters: competence, signal supply and paralogue group. Development 124: 849-859.

HOULE, M., SYLVESTRE, J.-R. and LOHNES, D. (2003). Retinoic acid regulates a subset of Cdx1 function in vivo. Development 130: 6555-6567.

ITASAKI, N., SHARPE, J., MORRISON, A. and KRUMLAUF, R. (1996). Reprogramming Hox expression in the vertebrate hindbrain: influence of paraxial mesoderm and rhombomere transposition. Neuron 16: 487-500.

KIECKER, C and NIEHRS, C. (2001). A morphogen gradient of Wnt/ $\beta$-catenin signalling regulates anteroposterior neural patterning in Xenopus. Development 128: 4189-4201.

KIM, M. H., SHIN, J. S., PARK, S., HUR, M-W., LEE, M-O., PARK, H. and LEE, CS. (2002). Retinoic acid response element in HOXA-7 regulatory region affects the rate, not the formation of anterior boundary expression. Int J. Dev. Biol. 46: 325-328.

KNITTEL, T., KESSEL, M., KIM, M. H. and GRUSS, P. (1995). A conserved enhancer of the human and murine Hoxa-7 gene specifies the anterior boundary of expression during embryonal development. Development 121: 1077-1088.

LICKERT, H., DOMON, C., HULS, G., WEHRLE, C., DULUC, I., CLEVERS, H., MEYER, B. I., FREUND, J-N. and KEMLER, R. (2000). Wnt/ $\beta$-catenin signaling regulates the expression of the homeobox gene $\mathrm{Cdx} 1$ in embryonic intestine. Development 127: 3805-3813

LIU, J-P., LAUFER, E. and JESSELL, T. M. (2001). Assigning the positional identity of spinal motor neurons: rostrocaudal patterning of Hox-c expression by FGFs, Gdf11 and retinoids. Neuron 32: 997-1012.

LOHNES, D. (2003). The Cdx1 homeodomain protein: an integrator of posterior signalling in the mouse. BioEssays 25: 971-980.

MARGALIT, Y., YARUS, S., SHAPIRA, E., GRUENBAUM, Y. and FAINSOD, A. (1993). Isolation and characterization of target sequences of the chicken CdxA homeobox gene. Nucleic Acids Res. 21: 4915-4922.

MEYER, B. I. and GRUSS, P. (1993). Mouse Cdx-1 expression during gastrulation. Development 117: 191-203.

OOSTERVEEN. T., NIEDERREITHER, K., DOLLÉ, P., CHAMBON, P., MEIJLINK, F. and DESCHAMPS, J. (2003). Retinoids regulate the anterior expression boundaries of 5' Hoxb genes in posterior hindbrain. EMBO J. 22: 262-269.

PILLEMER, G., EPSTEIN, M., BLUMBERG, B., YISRAELI, J. K., DE ROBERTIS, E. M., STEINBEISSER, H. and FAINSOD, A. (1998). Nested expression and sequential downregulation of the Xenopus caudal genes along the anteriorposterior axis. Mech. Dev. 71: 193-196. 
POWNALL, M. E., TUCKER, A. S., SLACK, J. M. W. and ISAACS, H.V. (1996). eFGF, Xcad3 and Hox genes form a molecular pathway that establishes the anteroposterior axis in Xenopus. Development 122: 3881-3892.

PÜSCHEL, A. W., BALLING, R and GRUSS, P. (1991). Separate elements cause lineage restriction and specify boundaries of Hox-1.1 expression. Development 112: 279-287.

SUBRAMANIAN, V., MEYER, B. I. and GRUSS, P. (1995). Disruption of the murine homeobox gene $\mathrm{Cdx} 1$ affects axial skeletal identities by altering the mesodermal expression domains of Hox genes. Cell 83: 641-653.

THEILER, K. (1989). The house mouse. Atlas of embryonic development. SpringerVerlag, N.Y.
VAN DEN AKKER, E., FORLANI, S., CHAWENGSAKSOPHAK, K., DE GRAAFF, W., BECK, F., MEYER, B. I. and DESCHAMPS, J. (2002). Cdx1 and Cdx2 have overlapping functions in anteroposterior patterning and posterior axis elongation. Development 129: 2181-2193.

VINCENT, J-P. and BRISCOE, J. (2001). Morphogens. Current Biology 11: R851-R854.
Received: April 2004 Reviewed by Referees: May 2004 Modified by Authors and Accepted for Publication: June 2004 